\title{
Characterization of Electron Orbital Angular Momentum Transfer to Nanoparticle Plasmon Modes
}

\author{
Tyler R. Harvey ${ }^{1}$, Jordan Chess ${ }^{1}$, Jordan S. Pierce ${ }^{1}$, Peter Ercius ${ }^{2}$, Benjamin J. McMorran ${ }^{1}$ \\ 1. Department of Physics, University of Oregon, Eugene, OR 97405, USA \\ 2. National Center for Electron Microscopy, Lawrence Berkeley National Laboratory, \\ Berkeley, CA 94720, USA
}

We observed the decay of an electron vortex beam from a state with orbital angular momentum $l=1 \hbar$ to $l=0$ by interaction with gold nanoparticle surface plasmon modes. Several optical studies have induced plasmon vortices using optical vortices and circularly polarized light and suggested their use in nanophotonic and plasmonic devices [1,2]. Direct observation of angular momentum transfer from electron vortices allows for unique identification of the orbital angular momentum associated with localized plasmon excitations down to the nanometer scale.

The electron vortex was produced in an FEI Titan transmission electron microscope (TEM) at $300 \mathrm{kV}$ with large spot size and strong gun lens to maximize the coherence of the beam. A forked diffraction grating (Fig. 1) is located in the condenser lens aperture of the microscope. The first diffracted order of the grating is focused to a nanoscale probe in the plane of the sample to be analyzed; this beam carries $1 \hbar$ orbital angular momentum [3]. In order to better characterize the interaction, we measured the intensity of scattering off of the nanoparticle (Fig. 6) as a function of beam radius.

The peak intensity of the vortex beam forms a ring (Fig. 2). Inside this ring, the intensity of the beam drops nearly to zero, as the azimuthal phase term of a vortex beam, $e^{i l \phi}$, is singular at the center of the beam. A gaussian beam with no angular momentum, however, has no phase singularity and the peak intensity is at the center of the beam. Thus, if an electron vortex beam with one unit of angular momentum transfers that angular momentum to a plasmon mode, the scattered component of the beam will be distinguishable as an increase in the intensity of the center of the beam.

This increase in the intensity of the center of the probe beam is exactly what we observed when the probe beam was concentric with an $18 \mathrm{~nm}$ gold particle (Fig. 5). A comparison of the integrated radial intensity of the beam off the nanoparticle and on the nanoparticle (Fig. 7) clearly shows an absolute increase in intensity in the center of the beam. This central intensity increase is visible even though a significant fraction of the incoming beam intensity is lost to high-angle scattering in interaction with the particle. The calculated peak scattered intensity is $16 \%$ of the original unscattered vortex beam peak intensity (Fig. 7). We also saw that the scattered intensity peaked as a fraction of total intensity when the beam radius was just smaller than the radius of the nanoparticle (Fig. 6). This peak interaction at the surface is strong evidence for interaction with plasmon states.

We have thus observed transfer of orbital angular momentum from an electron vortex to a gold nanoparticle. Further work with energy-filtered TEM can better identify the energy losses corresponding to transfer of orbital angular momentum. As forked diffraction gratings which generate beams with up to $100 \hbar$ angular momentum have been demonstrated [3], the technique we demonstrate here can be repeated for electron vorticies with any integer number of angular momentum. Thus, this technique makes possible the identification of localized plasmon modes of specified angular momentum on 
nanoparticles and nanostructures with high spatial resolution.

\section{References:}

[1] P. S. Tan et al., Appl. Phys. Lett. 92 (2008), p. 111108

[2] Y. Gorodetski et al., Phys. Rev. Lett. 101 (2008), p. 043903.

[3] B. J. McMorran et al., Science 331 (2011) p. 192.

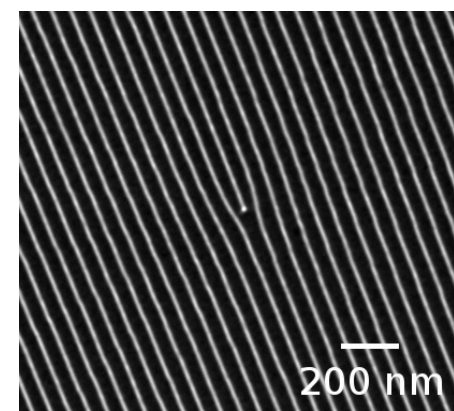

Figure

1 :

Transmission Electron Micrograph of the single-forked diffraction grating used to produce electron vortices with $\mathrm{n} \hbar$ orbital angular momentum in the nth diffraction order.

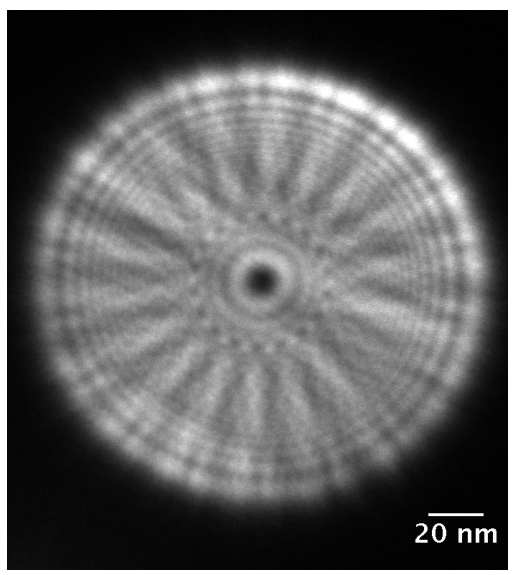

Figure 2: Micrograph of largeradius Electron Vortex on carbon substrate; taken under identical microscope conditions used to record the vortex-nanoparticle interaction.

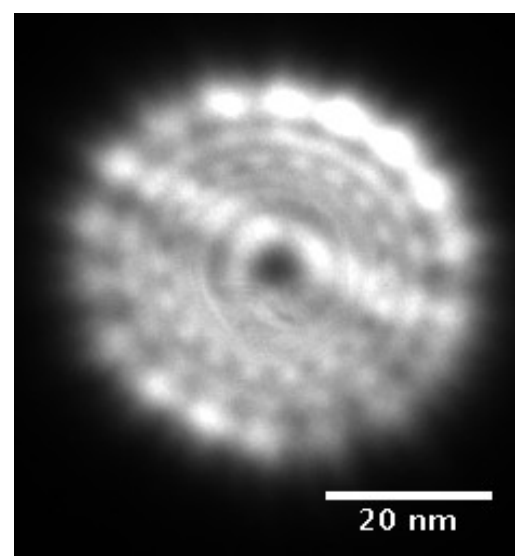

Figure 5: Micrograph of smallerradius Electron Vortex concentric on nanoparticle. A slight increase in intensity at the center of the beam is indicative of transfer of orbital angular momentum.

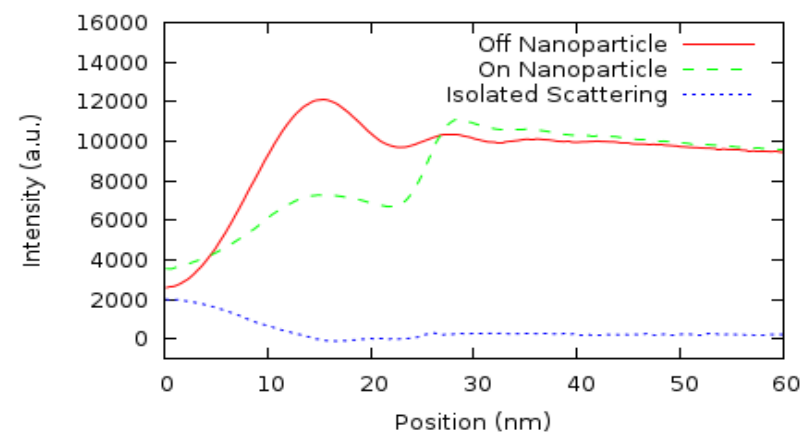

Figure 7: Comparison of the radial integrated intensity of the electron vortex off the nanoparticle (Fig. 4), on the nanoparticle (Fig. 5), and the isolated scattering due to the electron vortexnanoparticle interaction. 